\title{
Domains of Language Use: A Study of Burushos of Present day Kashmir
}

\author{
Sabba Mushtaq \\ University of Kashmir, Dept of Linguistics, Hazratbal, Srinagar, Kashmir, India
}

\begin{abstract}
The valley of Kashmir, being ethno-linguistically inordinately complex region, has accommodated different language speakers in its overall linguistic stratification. Apart from major languages like Kashmiri, Dogri, Ladakhi etc there are a multitude of minority languages like Burushaski, Shina, Balti etc. This paper will attempt to find out Burushaski language vitality among Burushos in Kashmir valley. It focuses on the domain analysis that is the domains where Burushaski is used and the domains where other languages are used. The paper is based on the questionnaires that the investigator took.
\end{abstract}

Keywords: Domain Analysis, Language Isolate, Burushaski, SPSS.

\section{Introduction}

Burushaski, also known by the names of Boorishki, Brugaski, Kanjut, Werchikwar and Mishaski is a language isolate spoken by some 87,000 Burusho people in Hunza, Nagar and Yasin area of Northern Pakistan. Linguistically, Burushaski has been termed as a language isolate because it does not characterize the genetic relationship with the surrounding language families like Indic, Sino-Tibetan, Dardic etc. Burushaski has been compared to almost every language on earth; no fully convincing relationships have yet been established. Modern taxonomic methods are, however, beginning to yield results. Ruhlen (1989) classified Burushaski as a language isolate: ,its genetic affiliation remains a complete mystery ${ }^{e e}$ but Ruhlen (1992) reports on a possible classification of Burushaski as a separate branch of a newly proposed Dene-Caucasian super stock. More recently, Blazek and Bengtson (1995) list tens of etymologies relating Burushaski to the Yeniseian languages, spoken by a hundred people along the Yenisei River in Siberia. None of these efforts has been accepted by scholarly consensus.

Burushaski speakers of Jammu and Kashmir are settled in and around a small locality by the foothills of Hariparbat in Srinagar, the capital of Jammu and Kashmir. The locality is known as Mohalla Azur Khan, named after Raja Azur Khan. Jammu and Kashmir Burushos are also reported to live in Tral (Pulwama), Batamaloo, and Dandusa in Bemina area of Srinagar. Jammu and Kashmir Burushos refer to their language as miśāski (mi-our + śāski-language).Exact number of the speakers of Jammu and Kashmir Burushaski is not available. According to the members of this speech community 300-350 Burushos live in Srinagar. Exact number of the speakers of Jammu and Kashmir Burushaski is not available. Munshi(2006) mentions that "Ethnologue (2005) mentions Burushaski speakers in India but does not provide about the specific information about the number of speakers and their exact location." According to the members of this speech community 300-350 Burushos live in Srinagar. The members of the Burushaski community in Srinagar form a tightly-knit subculture within the broader Kashmiri society. Almost all Burushos are related to each other. Burushos of Kashmir follow the Jaffaria School of thought. Most of the members of this community are the descendants of a tribal king who was originally from Nagar, Pakistan. It is for this reason that some members of the community claim to ascribe to a "higher" social status in terms of lineage, and while the rest are considered of a nonroyal descent by this sub-group. $\mathrm{J} \& \mathrm{~K}$ Burushos of the present day include some members who were originally from Hunza and probably migrated at a later stage. While Burushos enjoy a state domicile and have recently been offered a Scheduled Tribe status by the Government of India, in the state of Jammu \& Kashmir under Mandal commission. The migration of Burushos to Kashmir valley from Hunza and Nagar has taken place in 1891.This migration is the result of various political upheavals at these places. Certain historical accounts believe that this group migrated in intervals from 1891 onwards.

The linguistic repertoire of Jammu and Kashmir Burushos includes Burushaski, Balti, Kashmiri, Urdu and English. Language spoken at home is usually Burushaski. Balti, Kashmiri and Urdu are also used with varying degrees that is based on the linguistic proficiency of the individual and the social context in which they are used. The language that is used with the majority i.e. Kashmiri speakers is Urdu and Kashmiri. Being a highly educated community English is known to them. It is used as a language of communication for official and business purposes.

\section{Methodology}

The present study is based on the data collected from 76 language respondents during an intensive field work. The language respondents have been divided in social variables viz a) Age and b) Gender. Education although may be a significant social variable in some other studies but in the present study it has been observed that Burushos are educated across age and gender. The pilot survey has given the idea that education is not a relevant sociolinguistic variable to measure the issues related to the present study. The respondents were taken from Mohalla Azur Khan, Kathi Darwaza, Srinagar. All the respondents were the native speakers of Burushaski.

The questionnaire acted as a tool for eliciting the sociolinguistic data from the Burushos speech community. It consists of two sections. Each section has its own focus. 


\section{International Journal of Science and Research (IJSR) \\ ISSN (Online): 2319-7064}

Index Copernicus Value (2013): 6.14 | Impact Factor (2014): 5.611

Section I focused on discovering the background information of the subjects such as age, gender, education level and occupation.

Section II is aimed to obtain the data in terms of the language use in different domains: home, education, trade, work and, religion. It consists of 5 questions with subparts (Q1a,b,c,d,e, Q2a,b,c,d,Q3a,b,c,Q4a,b,c,Q5a,b). Under each of the 5 main domains, there are sub domains determined by interlocutor, activities and setting. After collecting the data from a survey instrument, the data was codified and then quantified. The quantitative data was captured in a software program called Statistical Package for the Social Sciences (SPSS) version 14.0 for its final analysis and tabulation purposes.

\section{Domain analysis}

The concept of domain was first proposed by Fishman as a way of looking at language choice. According to Fishman, domains are institutional context in which one language variety is more likely to be appropriate than another. Domains are constellation of factors such as location, activities, and participants. In this paper, language use of Burushos in five domains was examined. These domains were determined by interlocutor, activities and settings as shown in Table 1.1

Table 1.2: A Summary of Language Choice

\begin{tabular}{|c|c|c|c|c|c|c|c|c|c|c|c|}
\hline Question & $\mathrm{n}$ & Buru. & $\%$ & Bal & $\%$ & Kash. & $\%$ & Urd. & $\%$ & Eng & $\%$ \\
\hline \multicolumn{12}{|l|}{ 1. What do u speak at home With } \\
\hline \multirow{5}{*}{$\begin{array}{l}\text { a. Grandfather } \\
\text { b. Grandmother } \\
\text { c. Parents } \\
\text { d. Spouse } \\
\text { e. Children }\end{array}$} & 41 & 40 & 97.6 & 0 & & 0 & & 1 & 2.4 & 0 & \\
\hline & 54 & 51 & 94.4 & 2 & 3.7 & 0 & & 1 & 1.8 & 0 & \\
\hline & 74 & 68 & 91.9 & 0 & & 0 & & 6 & 8.1 & 0 & \\
\hline & 36 & 26 & 72.2 & 2 & 5.6 & 0 & & 8 & 22.2 & & \\
\hline & 34 & 29 & 85.3 & 0 & & 0 & & 5 & 14.7 & 0 & \\
\hline 2 a. With teacher inside the classroom & 74 & 0 & & 0 & & 6 & 8.1 & 60 & 81.1 & 8 & 10.8 \\
\hline b. With teacher outside classroom & 74 & 0 & & 0 & & 8 & 10.8 & 62 & 83.8 & 4 & 5.4 \\
\hline c. With friends in classroom & 74 & 0 & & 0 & & 22 & 29.7 & 52 & 70.3 & 0 & \\
\hline d. With friends outside classroom & 74 & 0 & & 0 & & 26 & 35.1 & 48 & 64.9 & 0 & \\
\hline \multicolumn{12}{|l|}{ 3.What language do you speak at market } \\
\hline With Burushaski speaker & 76 & 53 & 69.7 & 0 & & 0 & & 23 & 30.3 & 0 & \\
\hline With non-Burushaski & 76 & 0 & & 0 & & 0 & & 76 & 100 & 0 & \\
\hline With an acquaintance & 76 & 66 & 86.8 & 0 & & 0 & & 10 & 13.2 & 0 & \\
\hline \multicolumn{12}{|l|}{ 4. At work place } \\
\hline Talking to colleagues & 33 & 0 & & 0 & & 8 & 24.2 & 19 & 57.6 & 6 & 18.2 \\
\hline Talking with clients & 28 & 0 & & 0 & & 8 & 28.6 & 16 & 57.1 & 4 & 14.3 \\
\hline Talking with your leader & 33 & 0 & & 0 & & 3 & 9.09 & 20 & 60.6 & 10 & 30.3 \\
\hline \multicolumn{12}{|l|}{ 5. Language at Mosque } \\
\hline Asking God for something & 76 & 64 & 84.2 & 0 & & 0 & & 12 & 15.8 & 0 & \\
\hline Talking with other worshippers & 76 & 68 & 89.5 & 0 & & 0 & & 8 & 10.5 & 0 & \\
\hline Total & 1009 & 465 & 46.08 & 4 & 0.40 & 81 & 8.03 & 427 & 42.3 & 32 & 3.17 \\
\hline
\end{tabular}

As seen in the Table 1.2 the majority (465out of 1009) which is $46.08 \%$ of total responses sorted that Burushos use Burushaski language in most of the communication situations, such a large number of ,Burushaskie responses stand out clearly to indicate that the Burushaski language has a strong vitality.
Table 1.1: Domains of the Study

\begin{tabular}{|c|c|l|c|}
\hline Domain & Interlocutor & Activities & Settings \\
\hline Home & $\begin{array}{c}\text { Grandparents, Parents, spouse, } \\
\text { children, }\end{array}$ & & $\begin{array}{c}\text { In/out } \\
\text { classroom }\end{array}$ \\
\hline Education & Classmate and Teacher & & \\
\hline Market & $\begin{array}{c}\text { Burushaski/Non Burushaski, } \\
\text { acquaintances }\end{array}$ & & \\
\hline Workplace & Colleagues, Clients, Leaders & & \\
\hline Mosque & God, other worshippers & Praying & \\
\hline
\end{tabular}

In each domain, there are subdivisions with regard interlocutor and setting .e. $\mathrm{g}$ in the Home domain the interlocutors (grandparents, parents, spouse, and siblings) are listed as subdivisions of the Home domains.

\section{Language use in Five Domains}

In this paper 76 subjects were interviewed for their language use patterns in 5 domains. Table 1.2 is a summary of language choice for each domain of communication for the total research sample. The columns marked Burushaski, Balti, Kashmiri, Urdu and English etc indicated that the respondents reported that they use Burushaski, Balti, Kashmiri, Urdu and English to communicate in the situation. 


\section{International Journal of Science and Research (IJSR) \\ ISSN (Online): 2319-7064}

Index Copernicus Value (2013): 6.14 | Impact Factor (2014): 5.611

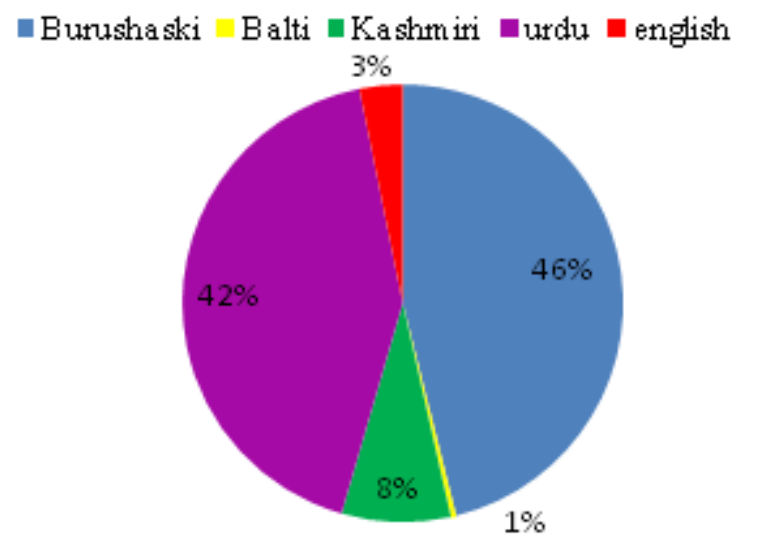

Figure 1.1: Pie graph of Percentage of Language choice in all domains (Total Sample)

The pie graph in fig. 1.1 shows the Burushaski has high dominance in communication in all domains. Regarding language use in each domain, among all 5 domains the choice of Burushaski stays very high. The average reported $\%$ of choice of Burushaski is always greater than the other languages which are used by the community as is shown in the above figure. The 5 domains and their corresponding questions are as following

1. Home(question $1 \mathrm{a}, \mathrm{b}, \mathrm{c}, \mathrm{d}, \mathrm{e})$

2. Education(question $2 \mathrm{a}, \mathrm{b}, \mathrm{c}, \mathrm{d}$, )

3. Market(question 3a,b,c,)

4. Workplace(question $4 a, b, c$, )

5. Mosque(question 5a,b,)

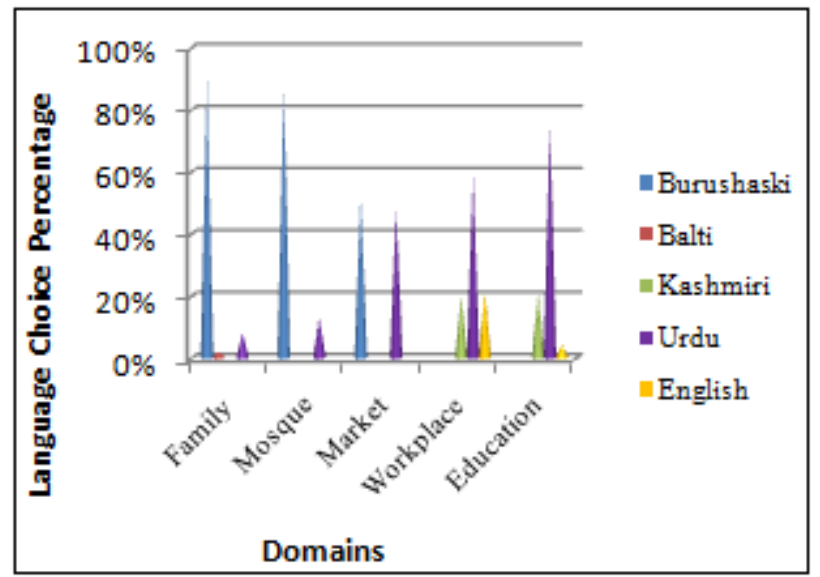

Figure 1.2: Language use in 5 domains
It can be seen from figure 1.2 that the use of Burushaski can be categorized into 2 levels $80 \%-90 \%$ and $50 \%-60 \%$. In the Home domain $90 \%$ of the respondents chose to use Burushaski in communication, over $80 \%$ but less than $90 \%$ of the respondents chose to use Burushaski in the mosque domain. In the market domain above $50 \%$ of the respondents use Burushaski. It can be seen from the above graph that the use of Burushaski in workplace and education domain stands nil and the use of Urdu/ Kashmiri / English is raised accordingly.

\section{Language use in the Home domain: (the in group Domain)}

Language use in the Home domain is investigated in almost every research concerning language maintenance and vitality. Some Studies have labeled family as a low domain in contrast with a high domain, while some treat it as informal domain contrast with a formal domain and thus refer to it as

An intimate domain vs. non intimate domain .Home domain is the most important domain for communication changes in language taking place in this domain may reflect changes happening elsewhere. When the language has lost battle in other domains, the Home domain often remains the last stand. Keep this domain and the language lives and lose it and the language dies.

In this paper, the language use of Burushaski in the Home domain stands out to indicate the strong language vitality of Burushaski.

Table 1.3 shows the summary of language use in the Home domain.

Table 1.3: Language use in Home Domain

\begin{tabular}{|c|l|c|c|c|c|c|c|c|c|c|c|c|}
\hline Q\# & Question & $\mathbf{n}=$ & Buru & \% & Bal. & \% & Kash & \% & Urd & \% & Eng. & $\%$ \\
\hline 1a & With Grand Father & 41 & 40 & $98 \%$ & 0 & & & & 1 & $2 \%$ & & \\
\hline 1b & With Grand mother & 54 & 51 & $94 \%$ & 2 & $4 \%$ & & & 1 & $2 \%$ & & \\
\hline 1c & With Parents & 74 & 68 & $92 \%$ & 0 & & & & 6 & $8 \%$ & & \\
\hline 1d & $\begin{array}{l}\text { With } \\
\text { Spouse }\end{array}$ & 36 & 26 & $92 \%$ & 2 & $6 \%$ & & & 8 & $2 \%$ & & \\
\hline 1e & $\begin{array}{l}\text { With } \\
\text { Children }\end{array}$ & 34 & 39 & $85 \%$ & 0 & & & & 5 & $15 \%$ & & \\
\hline
\end{tabular}

As we can see in Table 1.3 on an average over $90 \%$ of the subjects reported that they only speak Burushaski in the Home domain. The \% drops slightly when children are interlocutors. The reason for this shift is that some of the Burushos feel that learning other languages will fetch them bright future and attainment of prestigious jobs and better opportunities in life. Language use between generations has been the focus of various language studies. The change in the language use between generations indicates a likely ongoing language shift. It is shown in this study that $98 \%$ of the total 


\section{International Journal of Science and Research (IJSR) \\ ISSN (Online): 2319-7064}

Index Copernicus Value (2013): 6.14 | Impact Factor (2014): 5.611

subjects speak Burushaski to their grandfather, 94\% to their grandmother, $92 \%$ to parents, $92 \%$ to their spouses respectively. Balti as a language finds its place exclusively in home domain. $4 \%$ of the total respondent"s use Balti in this domain .Balti has been used in this community with Burushaski from the very beginning. Intermarriages with the females of Balti community were one of the reasons for incorporating this language among them. Burushos use Balti for communicating secret information or messages in presence of those who understand and know their language.

It is quite evident from the above table that Burushos do speak neither Kashmiri nor English in Home domain. When asked the researcher the common response was that Kashmiri and English can be learnt from other sources which being the e interaction with the majority speaking people i.e. Kashmiri, schools, exposure to mass and print media.

Overall, Burushaski is dominant language in the Home domain as can be seen in fig. 1.3.This indicates strong Burushaski vitality.

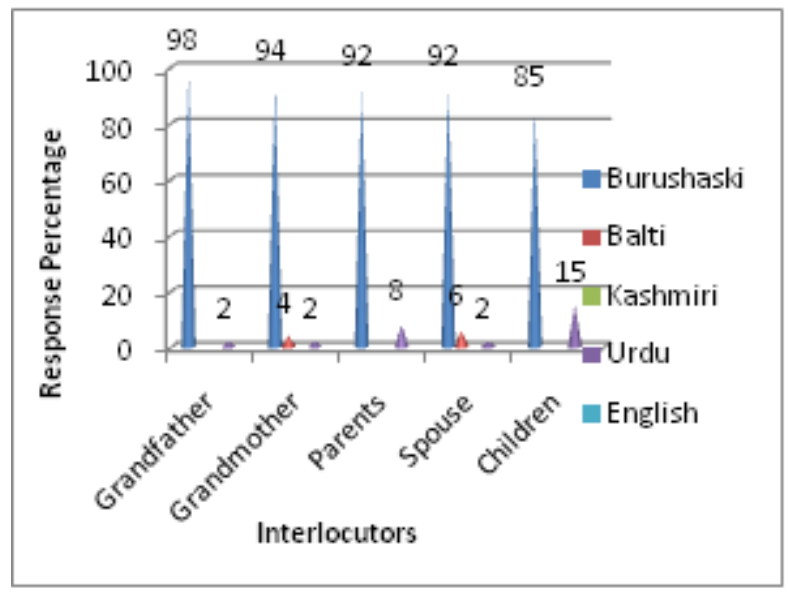

Figure 1.3: Language use in the Home domain
In Burushaski community, a shift to any other language does not reward one with high status and respect in turn but rather a bad evaluation from the in group people.

Language use in an unpredictable mixed group domain: In some other studies the mosque domain is placed at the other end of the continuum from the Home domain if a continuum of domains is ever used. In this study, the two domains government and mosque are grouped together and labeled as unpredictable mixed group domain. The term mixed group domain refers to the situation in which the initiator of the conversation cannot predict whether the interlocutor is a Burushaski speaker or not because in these domains both Burushaski and Kashmiri speakers exist. As a result one cannot easily predict which language should be used to talk to the interlocutor.

Table 1.4 is a summary of language use in Mosque domain:

Table 1.4: Language use in Mosque Domain

\begin{tabular}{|c|l|r|c|c|c|c|c|c|c|c|c|c|}
\hline Q\# & Question & $\mathrm{n}$ & Buru. & $\%$ & Bal. & $\%$ & Kash. & $\%$ & Urd. & $\%$ & Eng. & $\%$ \\
\hline 5a & What language you use while praying to Allah & 76 & 64 & $84 \%$ & & & & & 12 & $16 \%$ & & \\
\hline 5b & Talking with other worshippers? & 76 & 68 & $89 \%$ & & & & & 8 & $11 \%$ & & \\
\hline & Mean & $\mathbf{1 5 2}$ & $\mathbf{1 3 2}$ & $\mathbf{8 7 \%}$ & & & & & $\mathbf{2 0}$ & $\mathbf{1 3 \%}$ & & \\
\hline
\end{tabular}

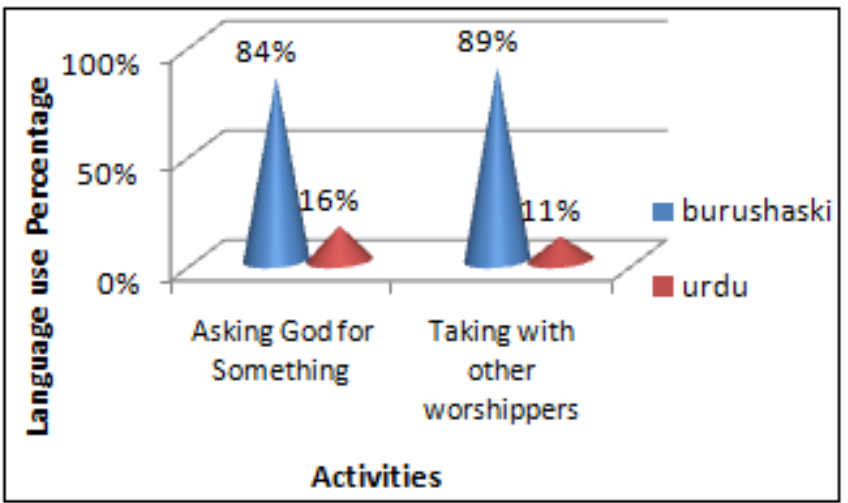

Figure 1.4: Language use in Mosque Domain

In this domain, as can be seen from Fig. 1.4 an average of more than $80 \%$ of the respondents chooses Burushaski as the only language of communication. Less than $20 \%$ of the respondents choose Urdu in their communication while Balti, Kashmiri and English are not used at all.

Based on the analysis above, it is clear that Burushaski is the dominant language in an un-predictable mixed group. This indicates a strong vitality of the Burushaski language.

\section{Language use in Predictable mixed group domain:-}

Education, market and workplace are grouped together as mixed group domain in which both Burushaski and Kashmiri speakers and Burushaski speakers exist. Different from the unpredictable mixed group domain, in the predictable mixed group domain, the initiator of the conversation can predict whether the Interlocutor is Burushaski or not or they know the particular norm for any topic or situation. . For example an employee should have knowledge about which language he is supposed to talk with the boss or colleagues. Table 1.5 is a summary of language use in these three domains:

\section{Volume 5 Issue 1, January 2016}




\section{International Journal of Science and Research (IJSR) \\ ISSN (Online): 2319-7064}

Index Copernicus Value (2013): 6.14 | Impact Factor (2014): 5.611

Table 1.5: Shows the Language use in Predictable mixed group domain

\begin{tabular}{|c|c|c|c|c|c|c|c|c|c|c|c|c|}
\hline Q\# & Question & $\mathrm{n}=$ & Buru. & $\%$ & Bal. & $\%$ & Kash. & $\%$ & Urd. & $\%$ & Eng. & $\%$ \\
\hline 2a & With teacher inside the classroom & 74 & 0 & & 0 & & 6 & $8 \%$ & 60 & $81 \%$ & 8 & $11 \%$ \\
\hline 2b & With teacher outside classroom & 74 & 0 & & 0 & & 8 & $11 \%$ & 62 & $84 \%$ & 4 & $5 \%$ \\
\hline 2c & With friends in classroom & 74 & 0 & & 0 & & 22 & $30 \%$ & 52 & $70 \%$ & 0 & \\
\hline 2d & With friends outside classroom & 74 & 0 & & 0 & & 26 & $35 \%$ & 48 & $65 \%$ & 0 & \\
\hline & Total & $\mathbf{2 9 6}$ & & & & & $\mathbf{6 2}$ & $\mathbf{2 1 \%}$ & $\mathbf{2 2 2}$ & $\mathbf{7 5 \%}$ & $\mathbf{1 2}$ & $\mathbf{4 \%}$ \\
\hline
\end{tabular}

\begin{tabular}{|c|c|c|c|c|c|c|c|c|c|c|c|c|}
\hline Q\# & Question & $\mathbf{n}=$ & Buru. & $\mathbf{\%}$ & Bal. & \% & Kash. & \% & Urd. & \% & Eng. & \% \\
\hline 3 & What language do you speak at market & & & & & & & & & & & \\
\hline a. & With Burushaski Speaker & 76 & 53 & $70 \%$ & & & & & 23 & $30 \%$ & 0 & \\
\hline b. & With non-Burushaski & 76 & 0 & & & & & & 76 & $100 \%$ & 0 & \\
\hline c. & With an acquaintance & 76 & 66 & $87 \%$ & & & & & 10 & $13 \%$ & 0 & \\
\hline & Total & $\mathbf{2 2 8}$ & $\mathbf{1 1 9}$ & $\mathbf{5 2 \%}$ & & & & & $\mathbf{1 0 9}$ & $\mathbf{4 8 \%}$ & & \\
\hline
\end{tabular}

\begin{tabular}{|c|c|c|c|c|c|c|c|c|c|c|c|c|}
\hline Q\# & Question & $\mathrm{n}=$ & Buru. & $\%$ & Bal. & $\%$ & Kash. & $\%$ & Urd. & $\%$ & Eng. & $\%$ \\
\hline 4 & At Work Place & & & & & & & & & & & \\
\hline a. & Talking to colleagues & 33 & & & & & 8 & $24 \%$ & 19 & $58 \%$ & 6 & $18 \%$ \\
\hline b. & Talking with clients & 28 & & & & & 8 & $29 \%$ & 16 & $57 \%$ & 4 & $14 \%$ \\
\hline c. & Talking with your leader & 33 & & & & & 3 & $9 \%$ & 20 & $61 \%$ & 10 & $30 \%$ \\
\hline & Total & 94 & & & & & 19 & $20 \%$ & 55 & $59 \%$ & 20 & $21 \%$ \\
\hline & Total of Question 2,3,4 & 618 & 119 & $19 \%$ & & & 81 & $13 \%$ & 386 & $63 \%$ & 32 & $5 \%$ \\
\hline
\end{tabular}

In these three domains, the language use of Burushaski dropped to a great extent comparing the use of other language such as Kashmiri, Urdu and, English as Fig. 1.5 shows. The use of Urdu language in these three domains is dominant followed by the use of Kashmiri and then by English.

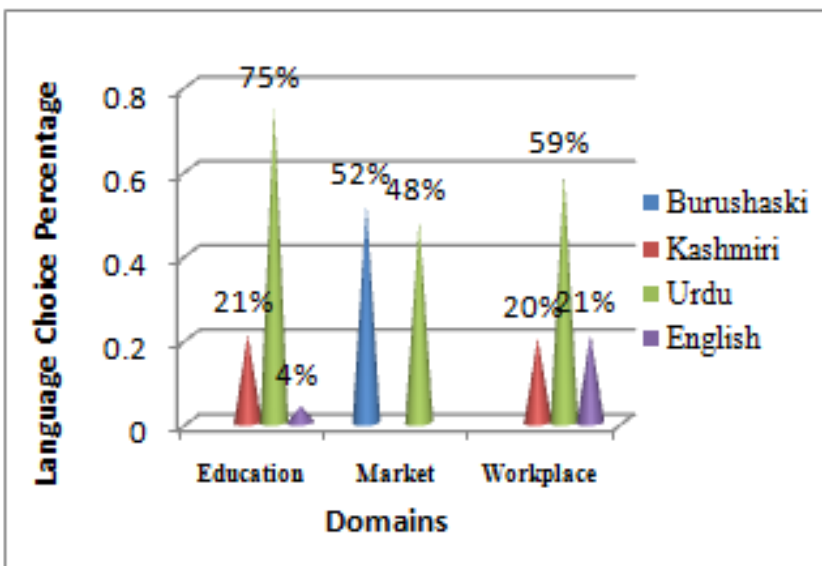

Figure 1.5: Language use in the predictable mixed group domain

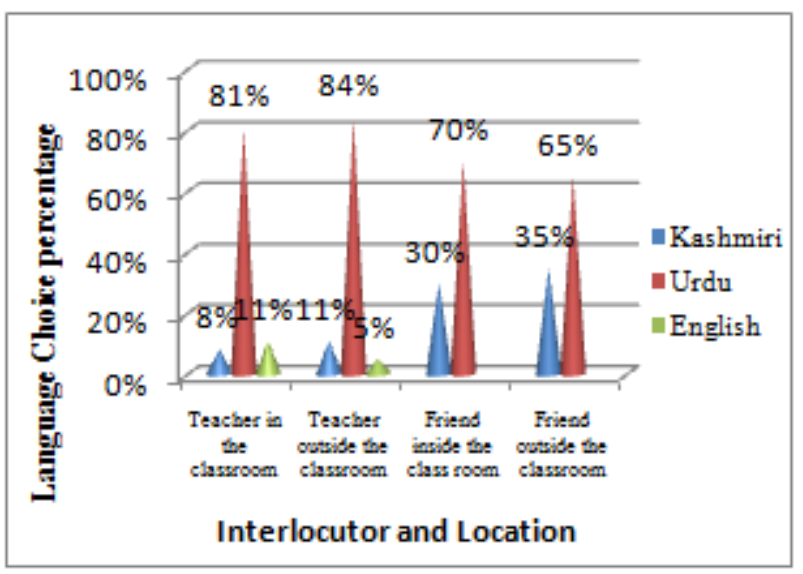

Figure 1.6: Language use in the Education Domain
In Education domain as fig. 1.6 shows the overall use of Urdu has a higher percentage in comparison to the other languages i.e. Kashmiri and English. The reason for such raise is that Urdu is the official language of the state and is used as medium of instruction in majority of the schools. As seen from the fig. 1.6 Kashmiri stands second on the scale because of the contact and influence of the majority speaking Kashmiri students on Burushaski speaking students in school. The environment of the school also can be attributed for the inclination towards the mainstream language i.e. Kashmiri. The preference for English language is relatively low because it is used only in Formal situations.

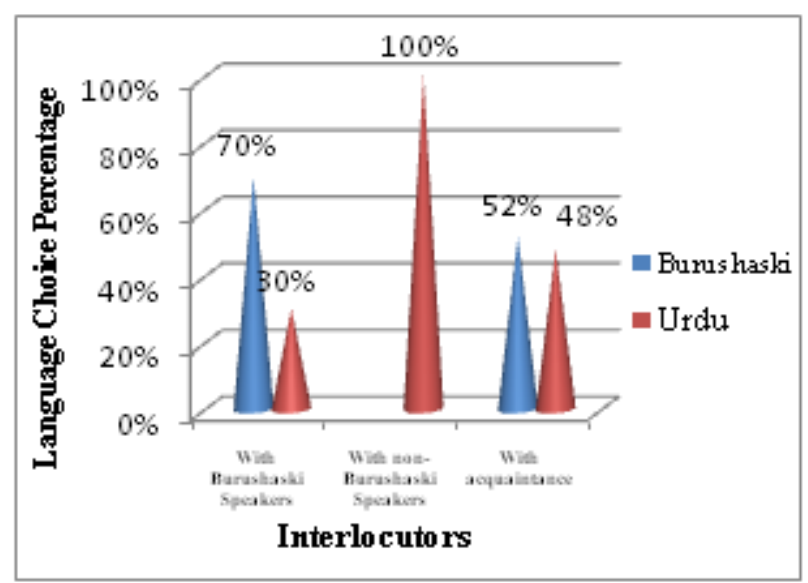

Figure 1.7: Language use in Market Domain

In Market domain only two languages as can be seen from fig. 1.7 were found to be used by the respondent's i.e. Burushaski and Urdu. With the Burushaski speakers in the market domain the respondents claimed to use Burushaski $(70 \%)$ and Urdu (30\%).Similarly with the non-Burushaski speakers only Urdu $(100 \%)$ is used. In case with acquaintances Burushaski (52\%) exceeds Urdu (48\%). Thus, from above data we can conclude that Urdu is used with all the three interlocutor groups. 


\section{International Journal of Science and Research (IJSR) \\ ISSN (Online): 2319-7064}

Index Copernicus Value (2013): 6.14 | Impact Factor (2014): 5.611

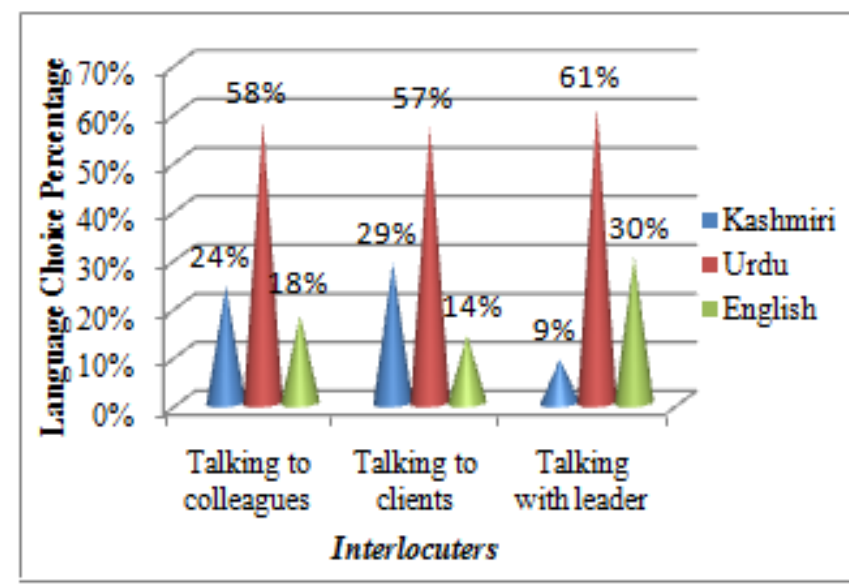

Figure 1.8: Language use in Workplace Domain

The workplace is the domain that exhibits the highest use of Urdu language. As figure 1.8 shows Urdu stays as the dominant language. On an average $60 \%$ of subjects choose Urdu to communicate in their work places being the official language of the state. Among the subjects of study nearly $30 \%$ use English in formal occasions and 5\% choose Kashmiri.

From the discussion above one can see that Burushaski is the dominant language in all the three groups of domains under investigation, although actual percentages are slightly different from domain to domain as fig. 1.9 shows

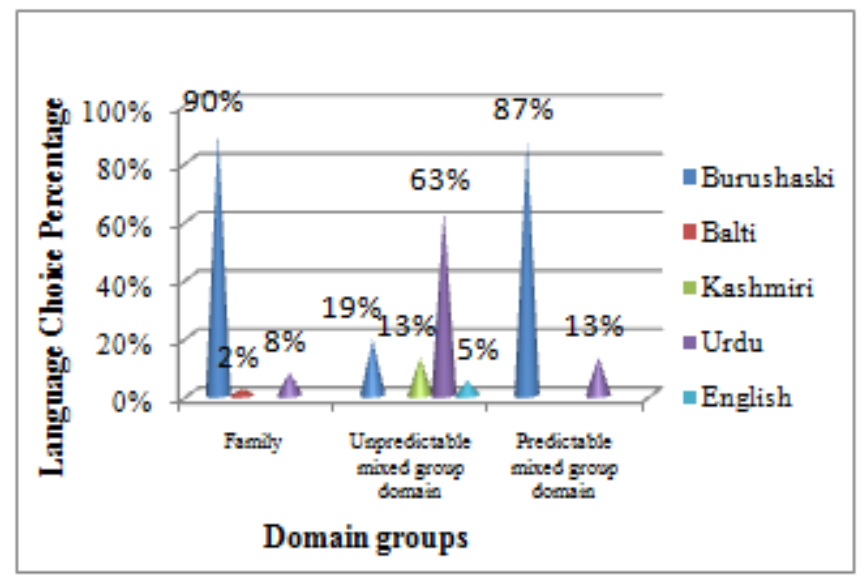

Figure 1.9: A Comparison of Language use in three Domain Groups

\section{Conclusions}

Language use in 5 domains was discussed respectively. According to the use of Burushaski 5 domains were grouped into 3 main domain groups:

a. The home domain

b. The unpredictable mixed group domain.

c. The predictable mixed group domain.

It was revealed that Burushaski language has absolute dominance in the home domain (fig. 1.3). Over $90 \%$ of the speakers speak Burushaski in home domain. This indicates strong vitality of Burushaski language. In the mixed group domain, the use of Burushaski is not as dominant as in the home domain (fig. 1.4 and 1.5). 19\% of the speakers reported that they use Burushaski in unpredictable mixed group domain while as in predictable mixed group domain use of Burushaski raises to $87 \%$. In the unpredictable mixed group domain the use of Burushaski declines drastically while as in predictable mixed group domain the use of Burushaski declines slightly. The use of other languages increased accordingly in both unpredictable and predictable mixed group domain. It is interesting to note that Balti strictly remains confined in the Home domain. However language use in all domains points towards one conclusion that Burushaski language vitality is very strong among Burushos in Kashmir valley.

\section{References}

[1] Colin Baker, Attitudes and Language, Multilingual Matters Ltd, Philadelphia, 1992.

[2] C. Butler, Statistics in Linguistics, Basil Blackwell, New York, 1985.

[3] Dick Grunes, Burushaski: An Extraordinary language in the Korakoram Mountains, Joseph Biddulph publishers, UK, 1998.

[4] G.A Grierson, Linguistic Survey of India, Volume X, Munshi Ram Manoharlal Publishers, Delhi, 1889.

[5] H.M. Batibo, Language decline and death in Africa: Causes, Consequences and Challenges, Multilingual Matters Ltd., Toronto, 2005.

[6] Michael C. Shapiro, \& Harold Schiffman, Language and Society in South Asia, Motilal Banarasidass Publishers Pvt.Ltd, New Delhi,1981.

[7] R. Fasold, The Sociolinguistics of Society, Basil Blackwell, New York, 1989.

[8] Sadaf Munshi, Jammu and Kashmir Burushaski: Language, Language Contact and Change, Ph.D Dissertation, University of Texas, Austin, 2006.

[9] Shahnaz Hunzia, Burushaski-Urdu Dictionary, Burushaski Research Academy, Hunza, 2009. 